\title{
Research Paper \\ Effect of 8-Week of Selected Aerobic Exercise on Static and Dynamic Balance in Healthy Elderly Inactive Men
}

\author{
*Masoud Mirmoezzi ${ }^{1}$, Mojtaba Amini ${ }^{2}$, Asghar Khaledan ${ }^{3}$, Davood Khorshidi ${ }^{4}$
}

1. Department of Biomechanics, Faculty of Physical Education and Sport Sciences, Central Tehran Branch, Islamic Azad University, Tehran, Iran.

2. Department of Physical Education and Sport Sciences, Faculty of Humanites and Social Sciences, Science and Research Branch, Islamic Azad University, Tehran, Iran. 3. Department of Physiology, Faculty of Physical Education and Sport Sciences, Karaj Branch, Islamic Azad University, Karaj, Iran.

4. Department of Physiology, Faculty of Physical Education and Sport Sciences, Saveh Branch, Islamic Azad University, Saveh, Iran.

Citation: Mirmoezzi M, Amini M, Khaledan A, Khorshidi D. [Effect of 8-week of selected aerobic exercise on static and dynamic balance in healthy elderly inactive men (Persian)]. Iranian Journal of Ageing. 2016; 11(4):202-209. http://dx.doi.org/10.21859/sija-1101202

: http://dx.doi.org/10.21859/sija-1101202

Received: 20 Oct. 2015 Accepted: 04 Feb. 2016

Key words:

Postural balance, Elderly, Aerobic exercise

\section{A B S TRACT}

Objectives Old age refers to years near or passed the middle age and therefore, near to the end of life cycle. Old-aged people have limited reviving capability and are more at risk of catching diseases, syndromes, and ailments. Falling is one of the most common problems in the elderly that occur due to their inability to maintain balance. This study aimed to examine the effect of aerobic exercises on static and dynamic balance in healthy elderly inactive men.

Methods \& Materials This quasi-experimental research was done in older people living in a nursing home in Qazvin, Iran. The study population included 200 elderly men (65-75 years old); of them, 26 healthy, inactive men were randomly selected and assigned to the experimental (13 subjects) and control (13 subjects) groups. The experimental group participated in selected exercise programs for 8 weeks. We used personal information questionnaire to check the inclusion criteria that comprised independency in doing daily chores, lack of problems in vision or fall during the last year, and not having joints dislocation, chronic arthritis or vertigo. Stork balance test and Timed Up and Go test were used to measure the static and dynamic balance of subjects before the initiation and after the termination of the program. The statistical analyses were done by paired and independent t-tests.

Results The results of independent t-test did not indicate any significant differences between 2 groups with regard to age, height, and weight $(P>0.05)$. Homogeneity was observed in the 2 groups with respect to individual features affecting balance and walking. After performing the aerobic exercise program, the dynamic balance in the experimental group increased significantly $(P<0.001)$. In the dynamic balance test, the lower mean time supported the better execution of the test, and significant changes were observed in the experimental group. The comparison of the two groups indicated the positive effect of aerobic exercise on the dynamic balance of healthy older people; however, no significant change was seen in static balance $(P=0.36)$. Since we used Stork test for determining static balance, the higher mean time supported the positive effect but with no significant change in the static balance of experimental group after 8 weeks. We found that different factors like lower extremity muscles such as soleus, gastrocnemius, and hamstring have some roles in keeping static balance, and as the exercise protocol was related to aerobic exercise (walking and jogging), it did not reinforce these muscles.

Conclusion Our results showed that regular aerobic exercise improves dynamic balance in elderly men. We also found that jogging and walking improved dynamic balance. The improvement in balance may be due to the increase in nervous compatibility (due to exercise); increase in fitness, strength, and motion domain; improvement in aerobic status, response time and mental status; improvement in neuromuscular control, including a decrease in changing movement units; and improvement in simultaneous movement units. Regular aerobic activities are recommended as a suitable training method for improving balance.

* Corresponding Author:

Masoud Mirmoezzi, PhD Candidate

Address: Department of Biomechanics, Faculty of Physical Education and Sport Sciences, Central Tehran Branch, Islamic Azad University, Tehran, Iran Tel: +98 (912) 6825007

E-mail: massoudmirmoezi@live.com 


\title{
تأثير ^هفته تمرين هوازى منتخب بر تعادل ايستا و يوياى مردان سالمند سالم غيرفعال
}

\author{
"مسعود ميرمعزى'، مجتبى امينى'، اصغر خالدان"، داود خورشيدى" \\ ا - كروه بيومكانيك، دانشكده تربيتبدنى و علوم ورزشى، واحد تهران مركز، دانشكاه آزاد اسلامى، تيهران، ايران.

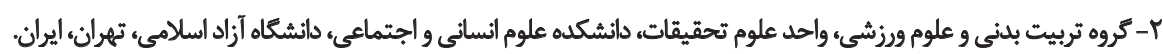

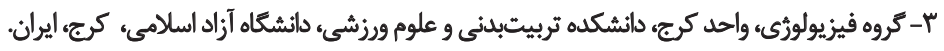

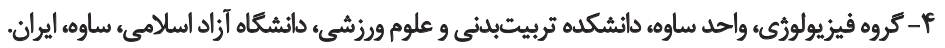

\begin{abstract}
حكبد

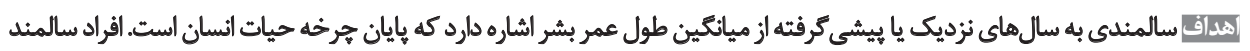

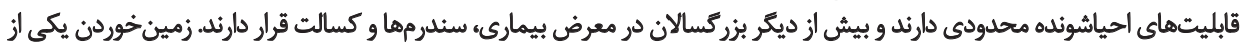

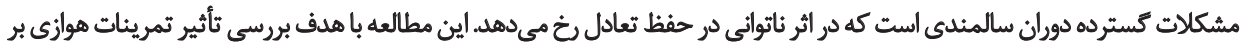

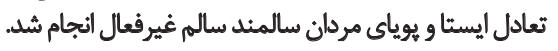

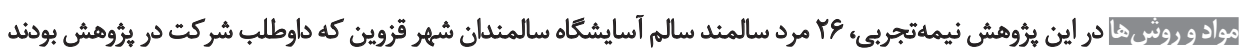

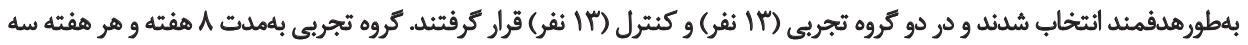

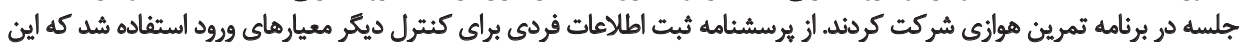

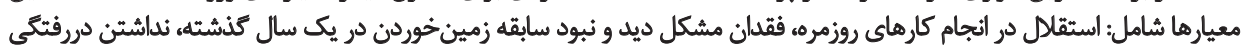

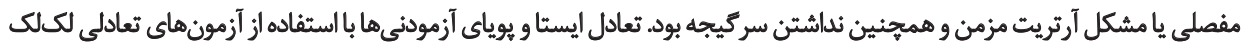

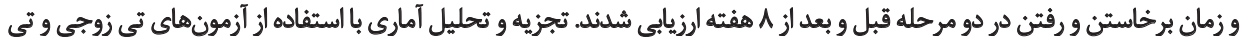
مستثمل انجام كرفت

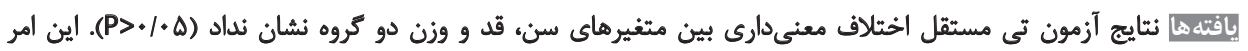

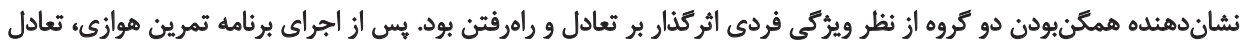

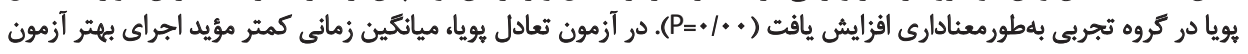

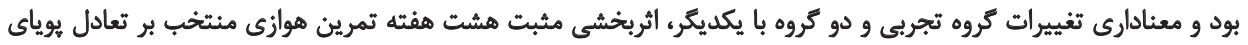

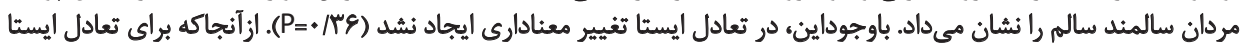

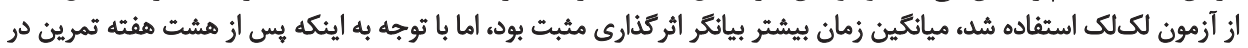

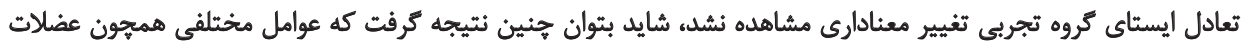

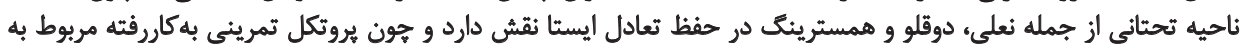

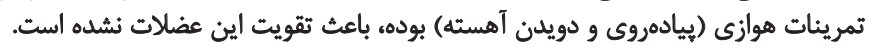

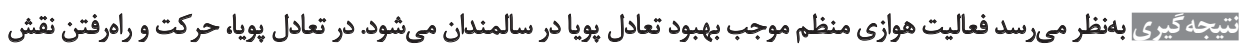

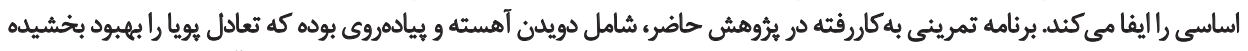

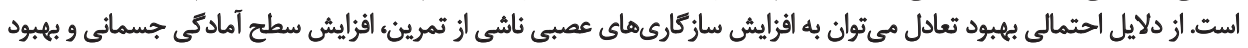

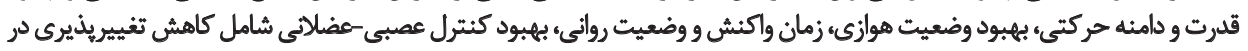

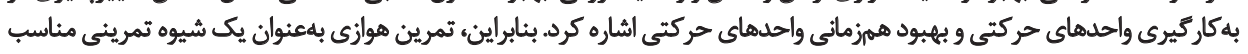

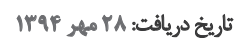

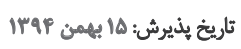


تحقيقات محدود در زمينه تأثير تمرينات هوازى بر تعادل سالمندان و

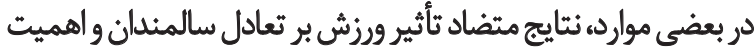

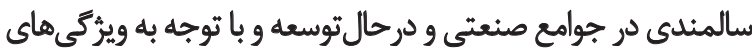

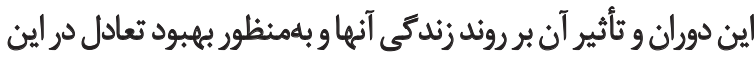

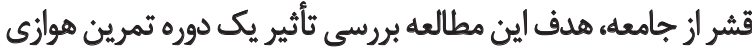
منتخب بر تعادل ايستاو يوياي مردان سالمند بودائ بردي

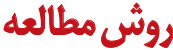

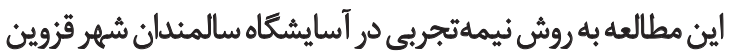

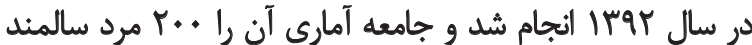

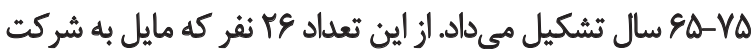

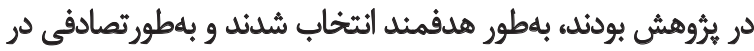

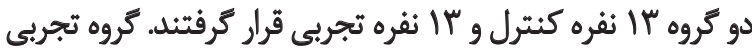

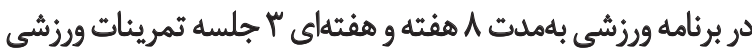

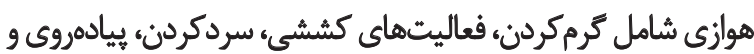

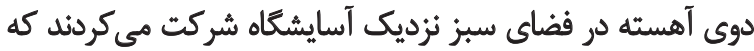

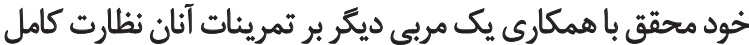

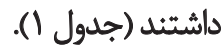

علاوهبراينهاز يرسشنامه ثبت اطلاعات فردى براى كنترل ديكر

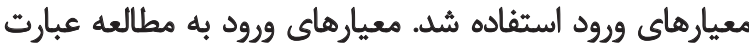

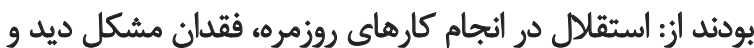

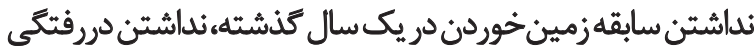

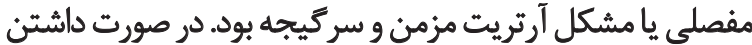

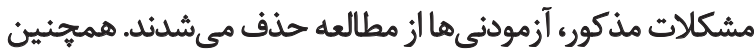

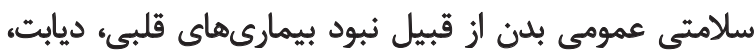

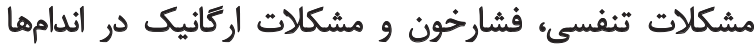

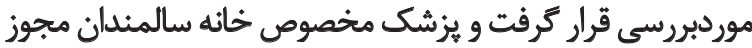

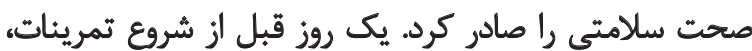
تعادل ايستاو يوياى آزمودنى ها اندازهيرى شئ شد.

تعادل ايستا توسط آزمون لكلك' كه سطح اعتبار آن /AV · بيان

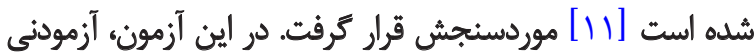

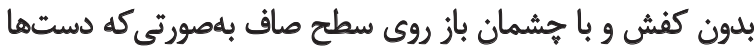

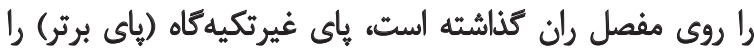

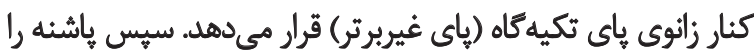

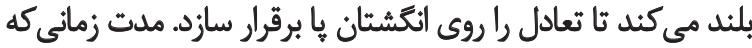

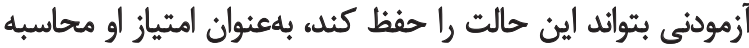

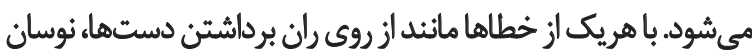

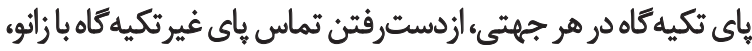

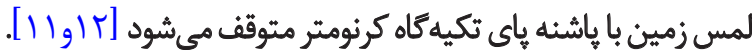
از آزمون زمان برخاستن و رفتن براى اندازهيرى تعادل يويا

1. Stork

2. Timed Get Up \& Go
با توجه به افزايش شاخص اميدبهزندگى در ايران براساس كَزارش

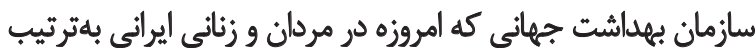

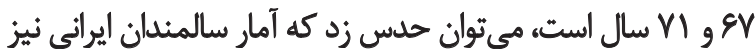

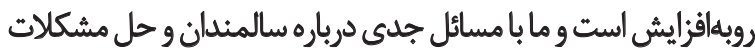

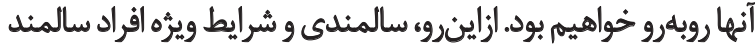
و تأمين بهداشت روانى و جسمانى آنها زاز جمله مسائلى است كه توجيه

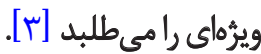

مهمثترين مسئله در ارتقاى سلامت سالمندان، مربوط به حفظ استقلال آنان در فعاليتهاى روزمره، كاركرد روزانه و شناخت ساخت

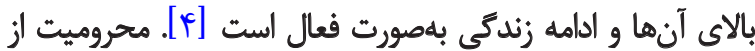

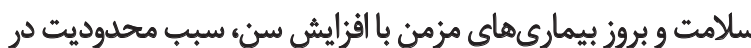

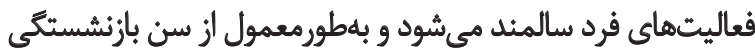

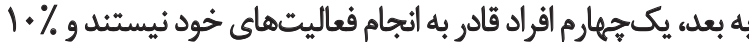

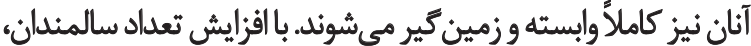

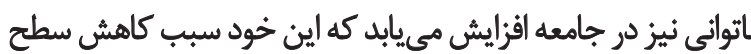

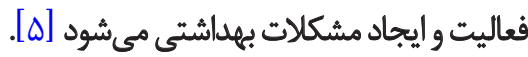
زمين بوردن نيز يكى از مشكلات شايع دوران سالمندى است

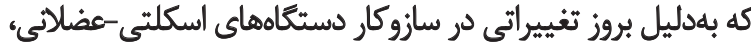

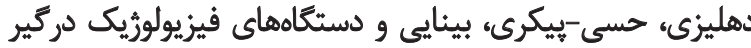

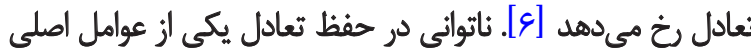

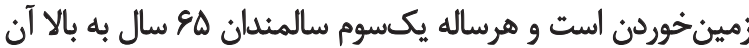

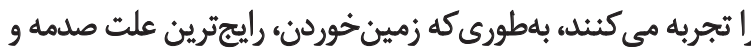

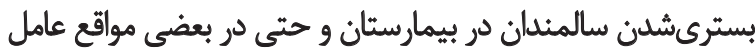

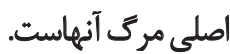

با افزايش سن، تعداد زمين خوردنها افزايش مىيابد كه عامل

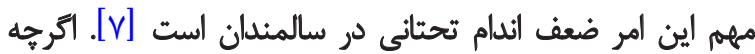

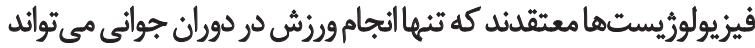

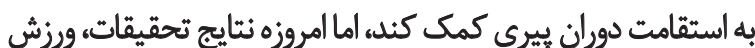

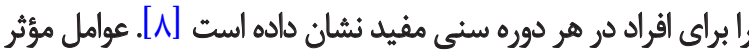

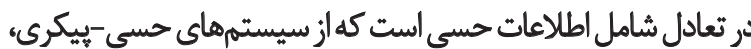

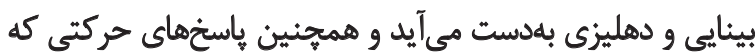

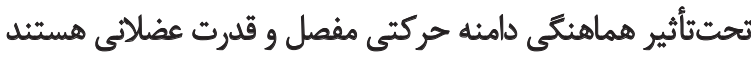

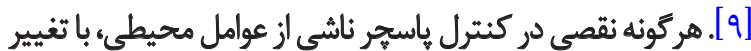

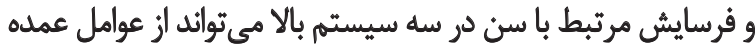

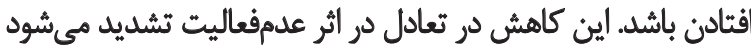

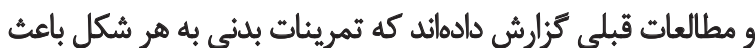

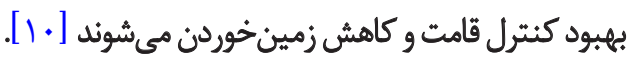
زمانى كه سالمندان احساس عدمتعادل و بـثباتى كنيند، ممكن

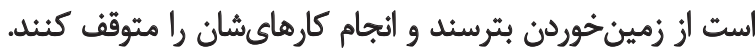

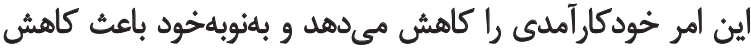

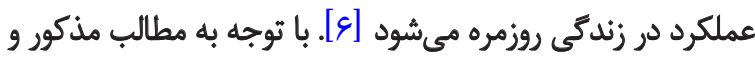


آزمون تى مسئقل استفاده شد. تجزيه و تحليل دادهها با استفاده از

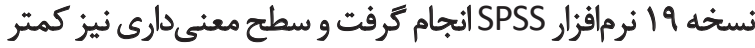

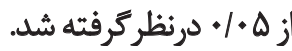

Lياقتهن

نتايج آزمون تى مستقل اختلاف معنى دارى بين متغيرهاى سن،

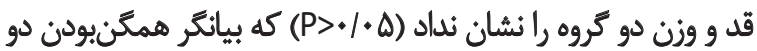

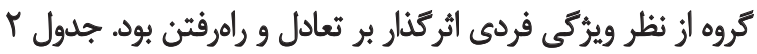

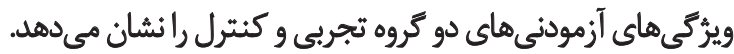
نتايج آزمون كلموكروف-اسميرنوف حاكى از طبيعىبودن توزيع

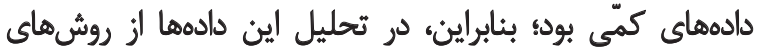

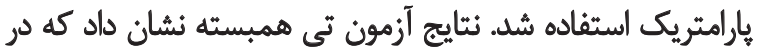

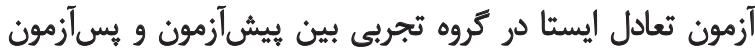

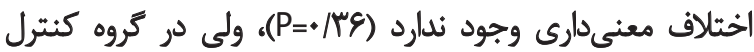

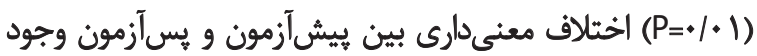

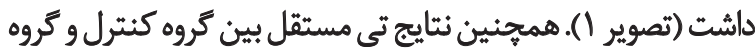

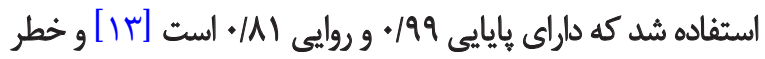

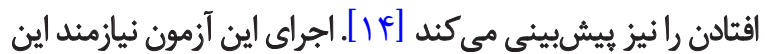

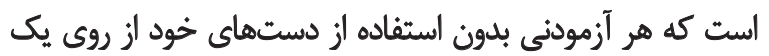

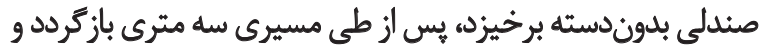

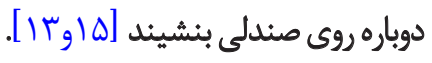

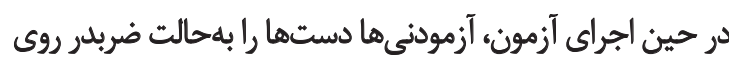

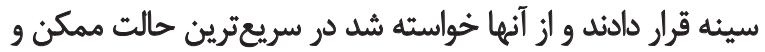

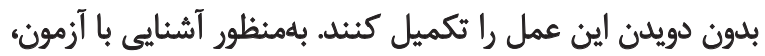

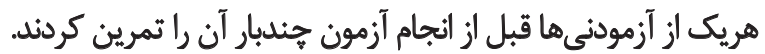

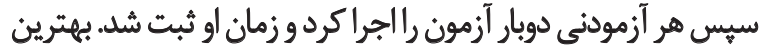

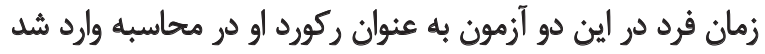

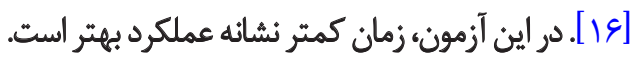
يس از تعيين طبيعىبودن توزيع دادهها با استفاده از آزمون

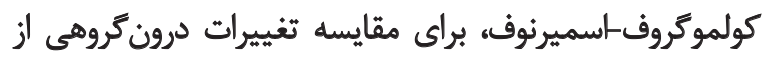

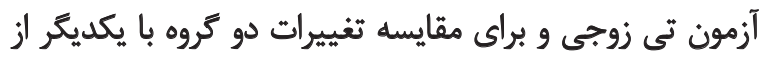

جدول ا. تمرينات ورزشى هوازى شامل ترمكردن، ئيادروى، دوى آهسته و سردكردن.

\begin{tabular}{|c|c|c|c|c|c|c|}
\hline مدت سردكردن & مدت آهستهدويدن & مدث بيادهروى & مدت كُمركردن & شدت تمرين & تعداد جلسات & هغته \\
\hline ما دمثيقه & r T بقيقه & ل 1 دقيقه & . إدقيقه & \% +ع ضربان قلب بيشينه & $r$ & 1 \\
\hline . & 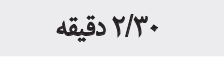 & 9 دقيقه & . الدقيقه & \% +ع ضربان قلب بيشينه & $r$ & r \\
\hline . 1 دقيقه & r ب بثيقه & . ال دقيقه & ا ا دقيقه & \% +8 ضربان ثلب بيشينه & r & $r$ \\
\hline ا دقيقه & . & ا & ا ادقيقه & 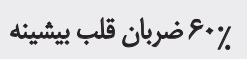 & r & f \\
\hline . 1 دقيقه & P أقيقه & ا I دقيقه & ا ا دقيقه & \% ه ضريان قلب بيشينه & $r$ & $\Delta$ \\
\hline ا دقيقه & . & "ا دقيقه & ا ادقيقه & \% \& ضربان قلب بيشيئه & $r$ & 8 \\
\hline مادقيقه & ه دقيقه & |f إقيقه & ا دقيقه & \% \$ صربان قلب بيشيئه & r & V \\
\hline ا دقيقه & . & ه 10 دقيقه & ا دقيقه & \% ض ضريان قلب بيشينه & $r$ & $\wedge$ \\
\hline
\end{tabular}

닌

جدول r. ويرَكى هاى آزمودنى ها به تفكيك كروه (انحراف استانداردثيانكين).

\begin{tabular}{|c|c|c|c|}
\hline وزن (كيلوكرم) & قد (سانتى متر) & سن (سال) & \\
\hline$e q / p a \pm r / 1 q$ & $\mid E N E P \pm Y / \cdot \Lambda$ & $e q / \Delta T \pm 1 / m$ & كروه تجربى \\
\hline ENTI \pm Tr/Tr & $V v \cdot / r \omega \pm r / F q$ & $e q / 4 T \pm 1 / 9 \Delta$ & كروه كتترل \\
\hline
\end{tabular}

ill

جدول ب. نتايج آزمون تى همبسته و مستمّل براى تعادل ايستا. 


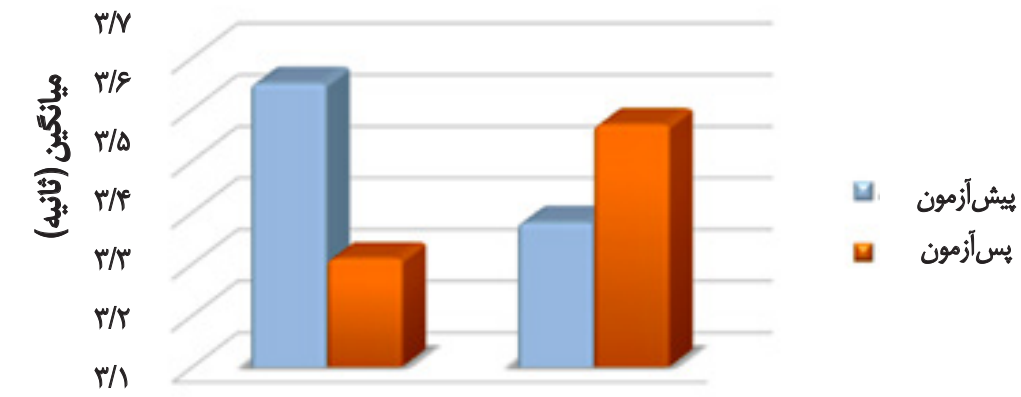

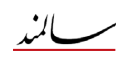

هوازى منتخب بر تعادل ايستا و يوياى مردان سالمند سالم

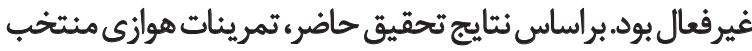

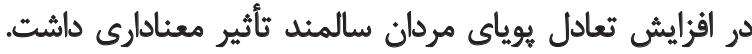

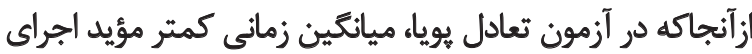

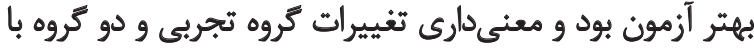

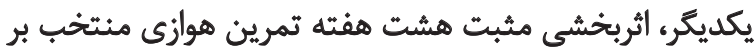

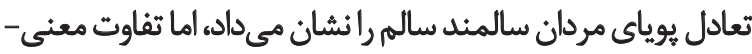
دارى در تعادل ايستاى مردان سالمئد ديده نشد.

در اين مطالعه براي تعادل ايستا از آزمون لكنلى استفاده شد

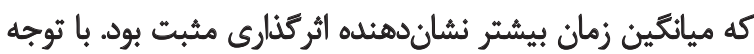

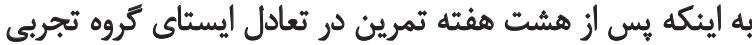

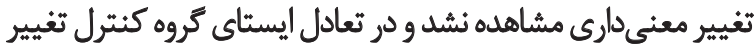

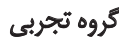

تصوير ا. مقايسه بيش آزمون و يسآزمون تعادل ايستا.

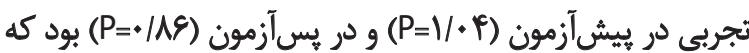
اختلافى مشاهده نشد (جدول بآ).

نتايج آزمون تى همبسته نشان داد كه در آزمون تعادل يوياء در

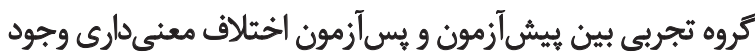

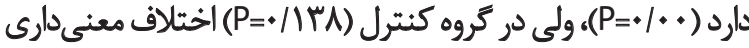

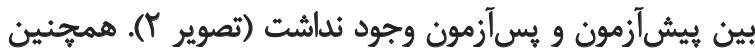

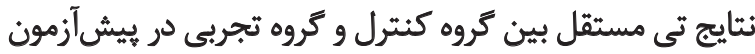

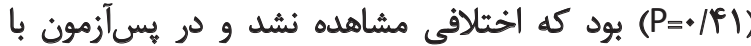
(P=॰/••) ب!

هدف اصلى تحقيق حاضر بررسى نأثير هشت هفته تمرين جدول P. نتايج آزمون نى همبسته و مستقل براى تعادل يويا.

\begin{tabular}{|c|c|c|c|}
\hline P-value & يسآزمون & ييش آزمون & \\
\hline . MrA & $\left|\Phi / F V_{ \pm}\right| /{ }^{\prime} \Lambda$ & $|\omega / \mu E \pm| / T F$ & كروه كتترل \\
\hline \multirow[t]{2}{*}{$10 *$} & $\mid r / g Y \pm \cdot / A \Psi$ & $|f|+r \pm \cdot / \lambda$ & كروه تجريى \\
\hline & $+1+0^{\circ}$ &.$/ 41$ & P-value \\
\hline
\end{tabular}

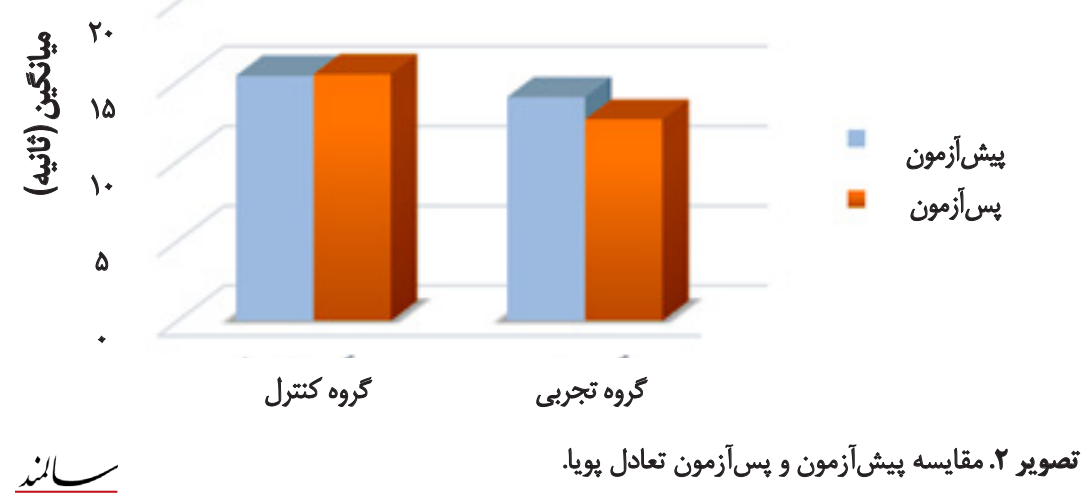




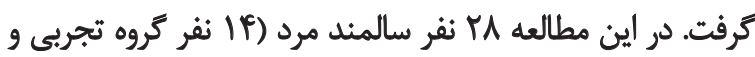

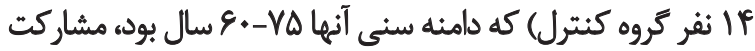

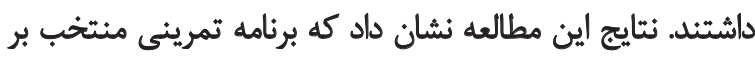

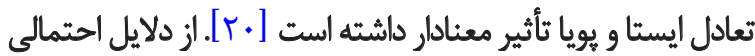

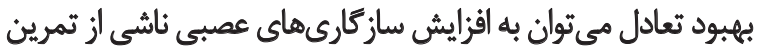

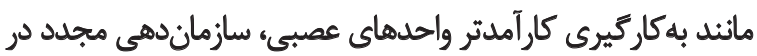

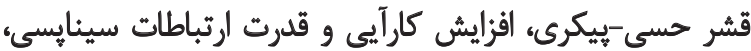

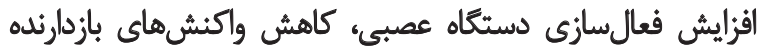

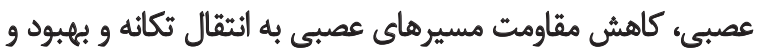

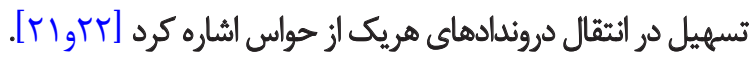
يكى از عوامل ديكر كه مى تواند توانايى افزايش تعادل و قدرت

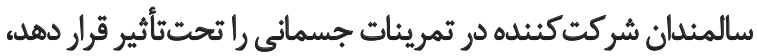

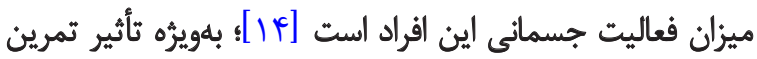

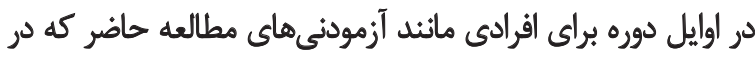

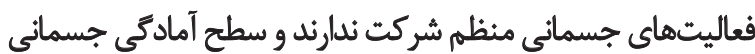
شان بايين است، مي تواند بيشتر باشد.

قدرت و دامنه حركتى از عوامل ديكرى است كه روى تعادل

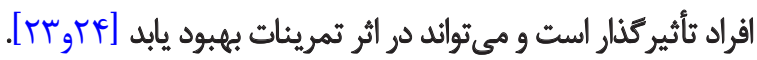

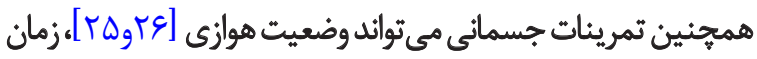

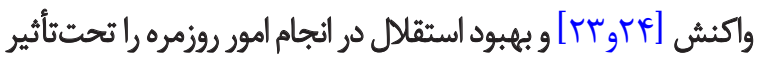

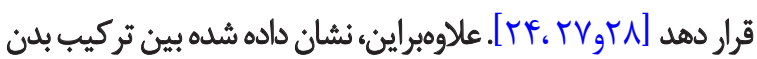

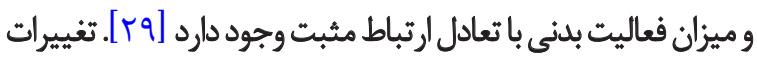

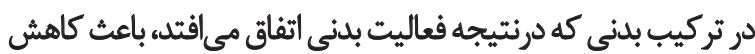

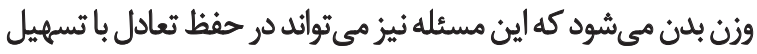

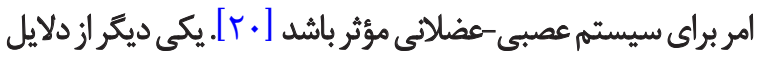

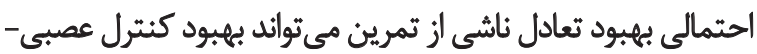

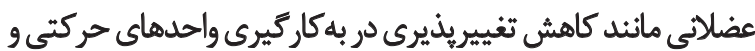

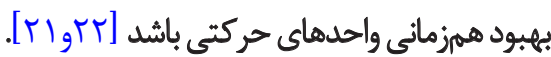

شايد ديكر عامل مؤثر در بهببود تعادل آزمودنىهائ اين مطالعه

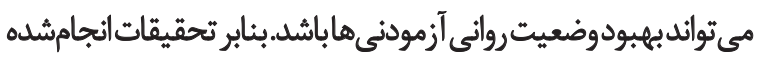
افزايش سن با افزايش ترس، اضطراب، استرس، افسردى آنى و كاهش

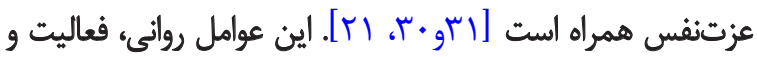

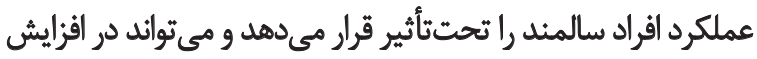

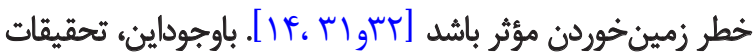

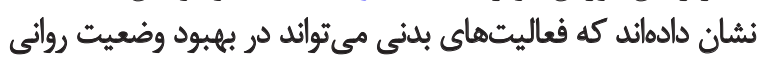

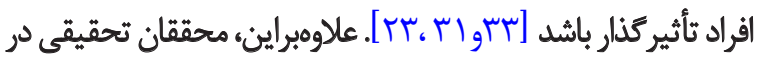

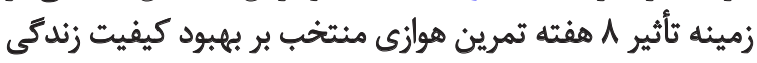

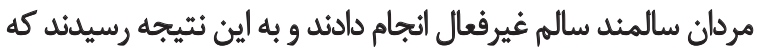

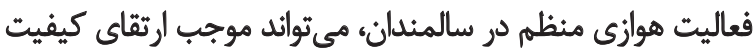

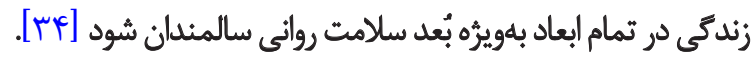

معنى دارى مشاهده شله شايد بتوان اينكونه نتيجه كيرى كرد كه

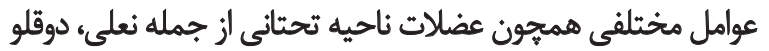

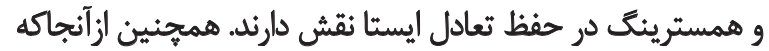

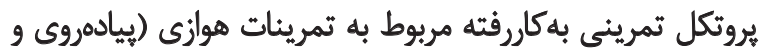

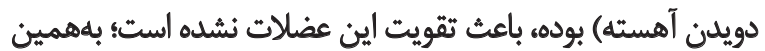

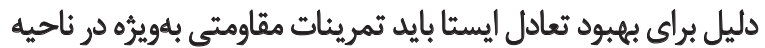

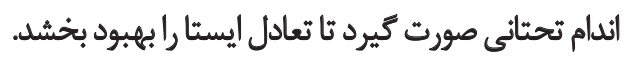

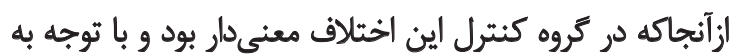

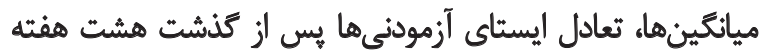

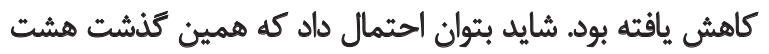

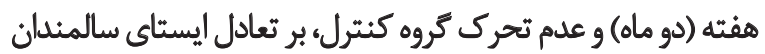

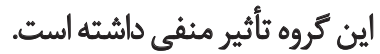

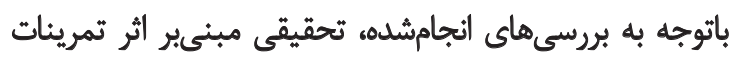

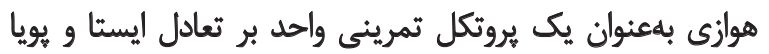

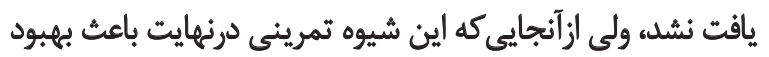

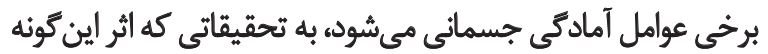

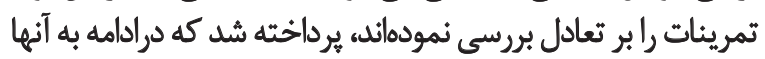

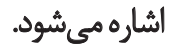

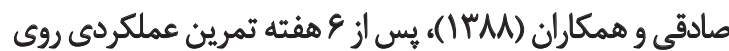

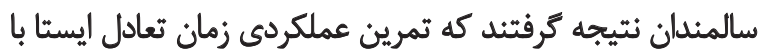

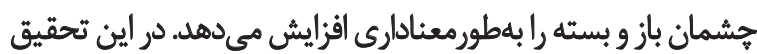

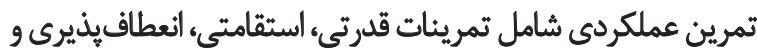

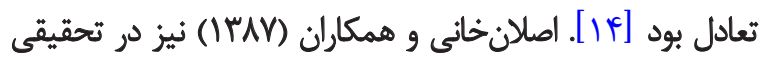

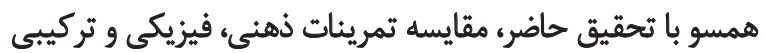

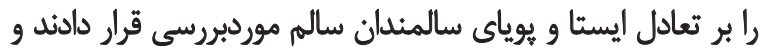

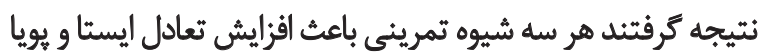

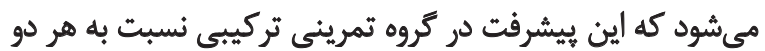

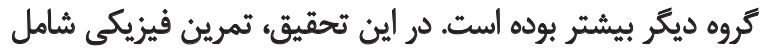

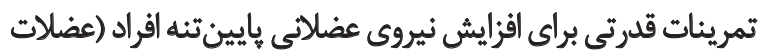

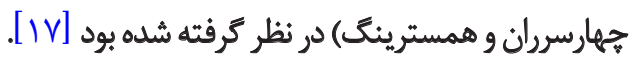

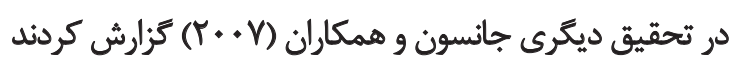

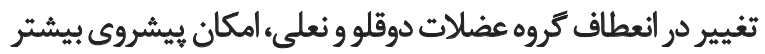

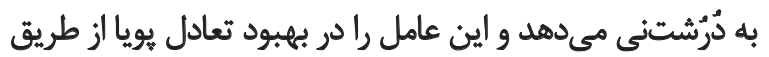

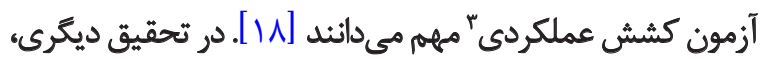

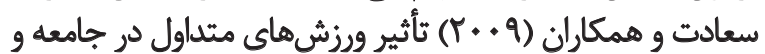

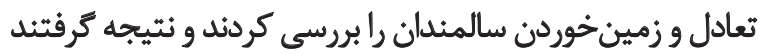

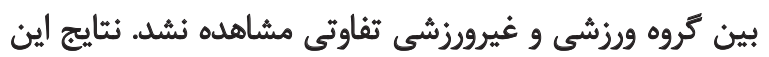
تحقيق بانتايج ديكر تحقيقات ناهمسو بورد تفرون

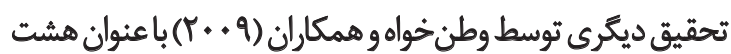

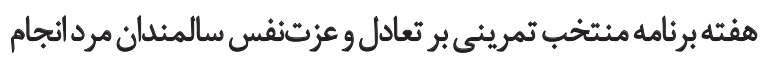




\section{References}

[1] Bernstein DA, Borkovec TD, Hazlett-Stevens H. New Directions in Progressive Relaxation Training: A Guidebook for helping professionals. California: Greenwood Publishing Group; 2000.

[2] Goldfried MR, Davison GC. Clinical behavior therapy. Hoboken, N.J.: Wiley \& Sons Publication; 1994.

[3] Shahbazzadegan B, Farmanbar R, Ghanbari A, Roshan ZA, Adib $M$. [The effect of regular exercise on self-esteem in elderly residents in nursing homes (Persian)]. Journal of Ardabil University of Medical Sciences. 2008; 8(4):387-393.

[4] Wood L, Peat G, Thomas E, Hay EM, Sim J. Associations between physical examination and self-reported physical function in older community-dwelling adults with knee pain. Physical Therapy. 2008; 88(1):33-42.

[5] McArdle WD, Katch FI, Katch VL. Physical activity, health and aging in exercise physiology: Energy, nutrition and human performance. $8^{\text {th }}$ ed. Baltimore: Williams and Wilkins Publication; 2014.

[6] Frändin K, Sonn U, Svantesson U, Grimby G. Functional balance tests in 76-year-olds in relation to performance, activities of daily living and platform tests. Scandinavian Journal of Rehabilitation Medicine. 1995; 27(4):231-41.

[7] Payne GV, Isaacs L. Human Motor Development: A Lifespan Approach. $8^{\text {th }}$ ed. New York: McGraw-Hill Education Publication; 2011.

[8] Schicht J, Camaione DN, Owen SV. Effect of intense strength training on standing balance, walking speed, and sit-to-stand performance in older adults. Journals of Gerontology, Series A: Biol ogical Sciences and Medical Sciences. 2001; 56(5):281-286.

[9] McAuley E, Elavsky S, Motl R, Konopack JF, Hu L, Marquez DX. Physical activity, self-efficacy, and self-esteem: Longitudinal relationships in older adults. Journals of Gerontology, Series B: Psychological Sciences. 2005; 60(5):268-275.

[10] Garshasbi A. [Compare the balance of active and inactive elderly men (Persian)] [MA thesis]. Tehran: Shahid Beheshti University; 2000.

[11] Johnson BL, Nelson JK. Practical measurements for evaluation in physical education. $4^{\text {th }}$ ed. Minneapolis: Burgess; 1979.

[12] Ogwumike OO, Tijani A. Balance performance of professional footballers with long-term lower limb musculoskeletal injury. African Journal of Physiotherapy and Rehabilitation Sciences. 2011; 3(1):23-7.

[13] Podsiadlo D, Richardson S. The timed "Up \& Go": A test of basic functional mobility for frail elderly persons. Journal of the American Geriatrics Society. 1991; 39(2):142-8.

[14] Sadeghi H, Norouzi HR, Karimi Asl A, Montazer MR. [Functional training program effect on static and dynamic balance in male able-bodied elderly (Persian)]. Iranian Journal of Ageing. 2008; 3(2):565-571

[15] Liu-Ambrose T, Khan KM, Eng JJ, Janssen PA, Lord SR, Mckay HA. Resistance and agility training reduce fall risk in women aged 75 to 85 with low bone mass: A 6-month randomized, controlled trial. Journal of the American Geriatrics Society. 2004; 52(5):657-65.

\section{نتيجه تيرى نهايى}

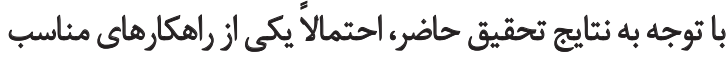

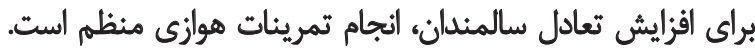

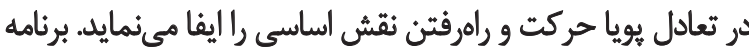

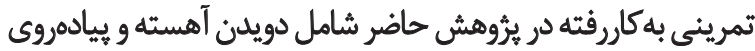

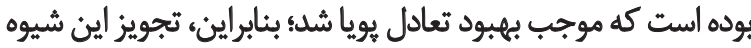
تمبينى باتوجه به اثرات آن بر ثعادل يويا به سالمئلان ثوصيه مىشود.

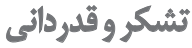

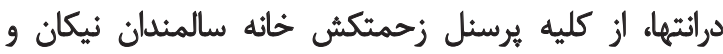

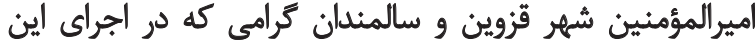

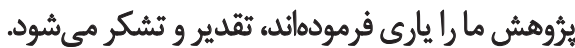


[16] Rogers ME, Rogers NL, Takeshima N, Islam MM. Methods to assess and improve the physical parameters associated with fall risk in older adults. Preventive Medicine. 2003; 36(3):255-264.

[17] Aslankhani MA, Shams A, Shamsipour P. [Comparison of mental exercises, and physical combination of static and dynamic balance in healthy elderly (Persian)]. Iranian Journal of Ageing 2008; 3(9-10):19-26.

[18] Johnson A, Shumway-cook A, Woollacatt MH. Motor control: Theory and practical applications. $3^{\text {rd }}$ ed. Baltimore: Williiams \& Willkins; 2007.

[19] Saadat Z. [Effect of exercise routine on the balance falls in the elderly (Persian)]. In: Hasani H, editor. Proceeding of the $16^{\text {th }}$ Congress of Iranian Occupational Therapy; 2009 Nov 19-20; Tehran, Ira. Availabe from: http://www.otci.ir/pic/Maghalat.pdf.

[20] Vatankhah A. [Effect of selected exercise program on balance and self-esteem in older men (Persian)]. In: Hasani H, editor. Proceeding of the $16^{\text {th }}$ Congress of Iranian Occupational Therapy; 2009 Nov 19-20; Tehran, Iran. Availabe from: http://www.otci. ir/pic/Maghalat.pdf.

[21] Stevens JA, Olson S. Reducing falls and resulting hip fractures among older women. Home Care Provider. 2000; 5(4):134-41.

[22] Dehkordi AS, Sokhangoei Y, Azarbayjani MA. Impact of an aquatic exercising program on the balance of elderly males. International Research Journal of Applied and Basic Sciences. 2012; 3(8):1678-1682.

[23] Shojaei M. [Motor Development (Persian)]. $2^{\text {nd }}$ ed. Tehran: Emam Hossein University; 2006.

[24] Hanai A, Yamamoto K, Hatakeyama T, Morita N, Okita K, Nomura T. Short-term water exercise effects on the physical fitness of elderly subjects from cold snowy region. Biomechanics and Medicine in Swimming. 2006; 6(2):363-5.

[25] Broman G, Quintana M, Lindberg T, Jansson E, Kaijser L. High intensity deep water training can improve aerobic power in elderly women. European Journal of Applied Physiology. 2006; 98(2):117-23.

[26] D'acquisto LJ, D'acquisto DM, Renne D. Metabolic and cardiovascular responses in older women during shallow-water exercise. Journal of Strength \& Conditioning Research. 2001; 15(1):1219.

[27] Chu KS, Eng JJ, Dawson AS, Harris JE, Ozkaplan A, Gylfadóttir S. Water-based exercise for cardiovascular fitness in people with chronic stroke: a randomized controlled trial. Archives of Physical Medicine and Rehabilitation. 2004; 85(6):870-4.

[28] Chandler JM, Duncan PW, Kochersberger G, Studenski S. Is lower extremity strength gain associated with improvement in physical performance and disability in frail, community-dwelling elders? Archives of Physical Medicine and Rehabilitation. 1998; 79(1):24-30.

[29] Hassinen M, Komulainen P, Lakka TA, Väisänen S, Rauramaa R. Associations of body composition and physical activity with balance and walking ability in the elderly. Journal of Physical Activity and Health. 2005; 2(3):298-306.

[30] Haywood K, Getchell N. Life span motor development with web resource. Champaign, I.L.: Human Kinetics; 2008.
[31] Gallahue D, Ozmun J. Understanding motor development: Infants, children, adolescents, adults with power web. New York, N.Y.: McGraw-Hill Humanities; 2005.

[32] Rosendahl E. Fall prediction and a high-intensity functional exercise program to improve physical functions and to prevent falls among older people living in residential care facilities. Sweden: Umea University Publication; 2006.

[33] Sohbatiha M, Rostamkhani H, Abbasi A, Gharaei E. [The effect of an aquatic exercise program on the quality of life of healthy elderly males: A comparative study (Persian)]. Journal of Research in Rehabilitation Sciences. 2010; 6(2):99-107.

[34] Amini M. Effect of 8-week of selected aerobic exercise on improving the quality of life and static and dynamic balance in healthy elderly inactive men (Persian)] [MA thesis]. Karaj: Karaj Islamic Azad University; 2014. 